\title{
PROPRIEDADES REDOX DE ÁCIDOS HÚMICOS ISOLADOS DE UM SOLO CULTIVADO COM CANA-DE-AÇÚCAR POR LONGO TEMPO ${ }^{(1)}$
}

\author{
Marihus Altoé Baldotto ${ }^{(2)}$, Luciano Pasqualoto Canellas ${ }^{(3)}$, \\ Maria Cristina Canela ${ }^{(4)}$, Carlos Eduardo Rezende ${ }^{(5)} \&$ Ary \\ Carlos Xavier Velloso ${ }^{(3)}$
}

\begin{abstract}
RESUMO
A matéria orgânica do solo é o maior reservatório de C nos sistemas naturais. Em tais sistemas a qualidade e a estabilidade do $\mathrm{C}$ podem ser estimadas pelo aumento da concentração das frações humificadas que, dentre outros fatores, está condicionada ao balanço entre as perdas e os ganhos que envolvem as reações de oxidação e de redução da matéria orgânica do solo. O objetivo deste estudo foi avaliar a eletroquímica, usando titulações redox iodimétricas, de ácidos húmicos isolados de solos cultivados continuamente com cana-de-açúcar submetida ou não à queima da palha para a colheita ou à adição anual de vinhaça. Os ácidos húmicos apresentaram valores do potencial formal padrão do eletrodo entre 0,760 e $0,779 \mathrm{~V}$ a $25^{\circ} \mathrm{C}$. A capacidade de oxidação dos ácidos húmicos variou de 1,01 a $3,44 \mathrm{~mol}_{\mathrm{c}} \mathrm{kg}^{-1}$ a pH 5,0 e de 1,64 a 6,44 $\mathrm{mol}_{\mathrm{c}} \mathrm{kg}^{-1}$ a $\mathrm{pH}$ 7,0. Observou-se correlação positiva e significativa entre a capacidade de oxidação dos ácidos húmicos e suas concentrações de grupos funcionais fenólicos, quinonas e semiquinonas.
\end{abstract}

Termos de indexação: química do solo, substâncias húmicas, potencial redox, capacidade de oxidação, grau de humificação.

\footnotetext{
(1) Parte da Tese de Doutorado do primeiro autor, apresentada à Universidade Estadual do Norte Fluminense Darcy Ribeiro UENF. Trabalho financiado pelo CNPq. Recebido para publicação em agosto de 2007 e aprovado em fevereiro de 2008.

(2) Pesquisador do Laboratório de Solos (LSOL) do Centro de Ciências e Tecnologias Agropecuárias (CCTA) da Universidade Estadual do Norte Fluminense - UENF. Av. Alberto Lamego 2000, CEP 28013-602 Campos dos Goytacazes (RJ). Bolsista do CNPq. E-mail: marihus@uenf.br

(3) Professor do LSOL do CCTA, UENF. Bolsista do CNPq. E-mails: canellas@uenf.br; velloso@uenf.br

(4) Professora do Laboratório de Ciências Químicas (LCQui) do Centro de Ciências e Tecnologias - CCT, UENF. Bolsista do CNPq. E-mail: mccanela@uenf.br

${ }^{(5)}$ Professor do Laboratório de Ciências Ambientais (LCA) do Centro de Biociências e Biotecnologia - CBB, UENF. Bolsista do CNPq. E-mail: crezende@uenf.br
} 


\title{
SUMMARY: REDOX PROPERTIES OF HUMIC ACIDS ISOLATED FROM A SOIL UNDER LONG-TERM SUGARCANE CULTIVATION
}

\begin{abstract}
Soil organic matter is the largest carbon reservoir in natural systems. In such systems the carbon quality and stability can be estimated based on the increase of humified fractions which, among other factors, is related to the balance between losses and accumulations involving oxidation and reduction reactions of the soil organic matter. The objective of this study was to evaluate the electrochemistry of humic acids isolated from soils cultivated continually with sugar-cane, with or without pre-harvest burning and annual vinasse application using iodimetric redox titrations. The formal electrode potentials of the humic acids had a similar pattern, with values between 0.760 and $0.779 \mathrm{~V}$, at $25^{\circ} \mathrm{C}$. The oxidation capacity of humic acids varied from 1.01 to $3.44 \mathrm{~mol}_{\mathrm{c}} \mathrm{kg}^{-1}$ at $\mathrm{pH} 5.0$ and from 1.64 to $6.44 \mathrm{~mol}_{\mathrm{c}} \mathrm{kg}^{-1}$ at $\mathrm{pH}$ 7.0. Positive and significant correlations were observed between the oxidation capacity and the humic acid contents of phenolic, quinone and semiquinone functional groups.
\end{abstract}

Index terms: soil chemistry, humic substances, redox potential, oxidation capacity, humification degree.

\section{INTRODUÇÃO}

O cultivo da cana-de-açúcar em Campos dos Goytacazez-RJ, nas proximidades do delta do Rio Paraíba do Sul, coincide com a própria história do Brasil. Em geral, áreas de Mata Atlântica cederam lugar aos canaviais. Dentre as estratégias de manejo dessa cultura, que também remetem a esse período de colonização, destaca-se, ainda hoje, a colheita usando-se o fogo, realizada para facilitar atividades de corte e carregamento da cana-de-açúcar. A manutenção da palha e a aplicação de resíduos da indústria sucroalcooleira (i.e., vinhaça) podem, por outro lado, beneficiar o solo e mitigar os efeitos nocivos da poluição atmosférica e da destinação dos resíduos da indústria canavieira, respectivamente.

As alterações nas propriedades de solos manejados com queima (cana queimada), preservação da palhada (cana crua) e aplicação de vinhaça foram estudadas por Canellas et al. (2003); Busato et al. (2005a,b) e Canellas et al. (2007). Os resultados desses trabalhos indicam que a preservação da matéria orgânica do solo (MOS) foi acompanhada de maior retenção e estabilidade de $\mathrm{C}$ no sistema, aumento da capacidade de troca de cátions (CTC) e dos teores de nutrientes, com concomitante diminuição do ponto de carga zero (PCZ).

No processo de humificação dos resíduos orgânicos, os ácidos húmicos (AH) representam a fração intermediária entre a estabilização de parte desses compostos pela interação com a matéria mineral (huminas) e a ocorrência de ácidos orgânicos na solução do solo (ácidos fúlvicos). Os AH são, portanto, um marcador natural da humificação dos resíduos orgânicos e refletem, como tal, tanto a condição de gênese, como de manejo do solo (Stevenson, 1994).
Com o avanço da humificação, ocorrem a policondensação e a conjugação de estruturas insaturadas nos AH (Piccolo, 2001). Grupamentos fenólicos formados durante o processo de decomposição da matéria orgânica são convertidos em quinonas, via reações de oxidação, as quais são precursoras de radicais livres do tipo semiquinonas nos $\mathrm{AH}$ (Milori et al., 2002; Budziac et al., 2004; Rivero et al., 2004), que se estabilizam com o avanço da humificação.

Alberts et al. (1974), Sunda \& Kieber (1994), Helburn \& MacCarthy (1994), Lovley et al. (1996, 1998), Scott et al. (1998) e Struyk \& Sposito (2001) mostraram que os $\mathrm{AH}$ possuem atividade redox em sistemas naturais. Baldotto et al. (2007) apresentaram medidas do potencial do eletrodo e da capacidade de oxidação de AH isolados de adubos orgânicos. Esses autores verificaram aumento da atividade redox de ácidos húmicos com o aumento da concentração de grupos funcionais fenólicos, quinonas e semiquinonas em sua estrutura, isto é, com o aumento de aromaticidade seletiva ou do grau de humificação. Para verificar a participação dos grupos funcionais nas reações redox, obtiveram derivados químicos metilados e acetilados de AH. A metilação dos $\mathrm{AH}$ com diazometano $\left(\mathrm{CH}_{2} \mathrm{~N}_{2}\right)$ converte grupos ácidos em $\mathrm{R}-\mathrm{OCH}_{3}$, denominados humatos de metila. Especificamente, grupos fenólicos e quinonas dos $\mathrm{AH}$ podem ser acetilados com anidrido acético em piridina, formando ésteres (Stevenson, 1994; Sachs et al., 2002). A titulação redox desses derivados metilados e acetilados mostra a participação dos grupos fenólicos e dos resíduos de quinonas nas reações redox que envolvem $\mathrm{AH}$.

O objetivo deste estudo foi avaliar a eletroquímica, usando as titulações redox iodimétricas em amostras de ácidos húmicos provenientes de solos cultivados continuamente com cana-de-açúcar. 


\section{MATERIAL E MÉTODOS}

\section{Área de estudo}

O solo em estudo, classificado como Cambissolo Ta Háplico eutrófico vértico (Embrapa, 1999), está localizado em Campos dos Goytacazes, no Estado do Rio de Janeiro (entre $41^{\circ} 00$ ' $-41^{\circ} 30^{\prime} \mathrm{W}$ e $21^{\circ} 00$ ' $-21^{\circ} 30^{\prime} \mathrm{S}$ ), em uma área plana e uniforme, $12 \mathrm{~m}$ acima do nível do mar. O material de origem consiste de aluvial franco-argiloso, proveniente de sedimentos neogênicos na bacia do Rio Paraíba do Sul, que compõe a formação denominada "Baixada dos Goytacazes" (Lamego, 1945). A média da precipitação pluvial ao longo dos anos em estudo foi de $1.080 \mathrm{~mm}^{-1}$, a temperatura média anual de $24,5^{\circ} \mathrm{C}$ e o clima é o Aw da classificação de Köppen.

As áreas em estudo se contrastam quanto ao manejo da cana-de-açúcar, ou seja, podem ser estudados dois casos de um longo tempo de cultivo. No primeiro deles, o cultivo foi realizado sem a utilização da queima da palha da cana-de-açúcar para o corte desde 1946 ("cana crua ou CC") e, no segundo caso, somente a partir de 1966, foi realizada a aplicação anual de $120 \mathrm{~m}^{3} \mathrm{ha}^{-1}$ ano $^{-1}$ de vinhaça via fertirrigação por aspersão ("com vinhaça ou CV”), totalizando, no momento da amostragem, em fevereiro de 2001, $55 \mathrm{e}$ 35 anos de manejo, respectivamente. Para comparação, foram amostrados talhões adjacentes, onde foi efetuada a queima da palhada da cana-deaçúcar para a colheita ("cana queimada ou CQ") e onde não foi aplicada vinhaça ("sem vinhaça ou SV"), respectivamente, ao longo dos mesmos períodos supracitados.

O manejo tradicional da cana-de-açúcar na região Norte Fluminense inclui o plantio de toletes em sulcos espaçados entre 1 e 1,5 m, controle químico de ervas daninhas e colheita manual. A reforma do canavial é realizada por meio de aração e gradagem, quando a produção atinge cerca de $70 \mathrm{t} \mathrm{ha}{ }^{-1}$, o que, geralmente, ocorre após o quarto corte. A adubação normalmente efetuada nessa região é de $60 \mathrm{~kg} \mathrm{ha}^{-1}$ de N, $60 \mathrm{~kg} \mathrm{ha}{ }^{-1}$ de $\mathrm{P}_{2} \mathrm{O}_{5}$ e $60 \mathrm{~kg} \mathrm{ha}^{-1}$ de $\mathrm{K}_{2} \mathrm{O}$, de uma única vez, no plantio. Como os quatro talhões têm morfologia semelhante, pois fazem parte de área onde todas as outras práticas de manejo são uniformes (de acordo com os depoimentos dos proprietários entrevistados), essas áreas podem ser comparadas por diferenças induzidas por práticas de manejo em longo prazo.

\section{Amostragem do solo e isolamento dos ácidos húmicos}

Na amostragem do solo, três parcelas de 0,05 ha constaram do delineamento inteiramente ao acaso para cada uma das quatro áreas em estudo. Para cada caso, o solo foi amostrado em duas profundidades (0,00-0,20 e 0,20-0,40 m), coletando-se ao acaso, com trado de $5 \mathrm{~cm}$ de diâmetro, 10 amostras simples em cada parcela, que resultaram em uma amostra composta por repetição.
Os AH foram isolados dos adubos orgânicos, conforme as recomendações da Sociedade Internacional de Subtâncias Húmicas (IHSS, 2006), usando-se $\mathrm{NaOH}$ 0,1 mol L-1 sob atmosfera de $\mathrm{N}_{2}$. Após agitação por $24 \mathrm{~h}$, o material foi centrifugado a $5.000 \mathrm{~g}$ por $30 \mathrm{~min}$. O sobrenadante foi coletado e o $\mathrm{pH}$ do extrato foi imediatamente ajustado a $1,5 \mathrm{com} \mathrm{HCl} 6 \mathrm{~mol} \mathrm{~L}^{-1}$. Depois de 18 h, a fração ácidos fúlvicos foi sifonada e descartada, permanecendo no frasco o precipitado de AH. A dissolução e a reprecipitação dos AH foram repetidas por mais duas vezes. A seguir, o precipitado de $\mathrm{AH}$ foi solubilizado e permaneceu em $\mathrm{HF}+\mathrm{HCl}$ $5 \%$ por $48 \mathrm{~h}$, visando à remoção de resíduos de minerais de argila e de íons paramagnéticos, sendo, a seguir, centrifugado a $5.000 \mathrm{~g}$. Os AH foram lavados com $200 \mathrm{~mL}$ de $\mathrm{HCl} 0,01 \mathrm{~mol} \mathrm{~L}^{-1}$, centrifugados a $5.000 \mathrm{~g}$. A seguir, o precipitado de $\mathrm{AH}$ foi lavado com água destilada até teste negativo para $\mathrm{Cl}^{-}$, usando-se $\mathrm{AgNO}_{3}$ 0,1 mol L-1, e, depois, transferido para membranas de diálise de $10 \mathrm{~mL}$ (cut-off $14 \mathrm{KDa}$, Thomas Sci.). Após a diálise, até à obtenção de condutividade elétrica igual à da $\mathrm{H}_{2} \mathrm{O}$ destilada $\left(1,0 \mu \mathrm{S} \mathrm{cm}^{-1}\right)$, os $\mathrm{AH}$ foram liofilizados e armazenados em dessecador.

Algumas propriedades químicas do solo e do $\mathrm{AH}$ (Quadro 1) foram previamente estudadas por Canellas et al. (2003, 2007).

\section{Determinação dos grupos funcionais e obtenção de derivados acetilados e metilados de ácidos húmicos}

A determinação dos grupos funcionais ácidos dos AH foi realizada de acordo com Schnitzer \& Gupta (1965). Foram determinadas a acidez total e a carboxílica experimentalmente e, pela sua diferença, a acidez fenólica. Para determinar a acidez total, foram adicionados, em um frasco Erlenmeyer de $125 \mathrm{~mL}, 100 \mathrm{mg}$ de $\mathrm{AH}$ e $20 \mathrm{~mL} \mathrm{Ba}(\mathrm{OH})_{2}$ 0,125 $\mathrm{mol} \mathrm{L}^{-1}$. Simultaneamente, foi preparada uma amostra em branco. $\mathrm{O}$ ar do frasco foi trocado por $\mathrm{N}_{2}$ através do borbulhamento por 5 min e o sistema foi agitado por $24 \mathrm{~h}$ à temperatura ambiente $\left(25^{\circ} \mathrm{C}\right)$. A seguir, a suspensão foi filtrada e o resíduo foi lavado com água destilada livre de $\mathrm{CO}_{2}$ (água fervida e resfriada para $\left.25^{\circ} \mathrm{C}\right)$. O excesso de $\mathrm{Ba}(\mathrm{OH})_{2}$ da suspensão foi titulado potenciometricamente com $\mathrm{HCl} 0,5 \mathrm{~mol} \mathrm{~L}^{-1}$ até $\mathrm{pH} 8,4$. A extração da acidez carboxílica (teor de grupos $\mathrm{COOH}$ ) foi realizada com acetato de cálcio e determinada por titulação com hidróxido de sódio. Em um frasco Erlenmeyer de $125 \mathrm{~mL}$, foram adicionados $100 \mathrm{mg}$ de $\mathrm{AH}, 10 \mathrm{~mL}$ de $\mathrm{Ca}(\mathrm{OAc})_{2} 1,0 \mathrm{~mol} \mathrm{~L}^{-1}$ e $40 \mathrm{~mL}$ de água destilada livre de $\mathrm{CO}_{2}$. Concomitantemente, foi preparado o teste em branco. Sob atmosfera de $\mathrm{N}_{2}, \mathrm{O}$ sistema foi agitado por $24 \mathrm{~h}$ à temperatura ambiente. A seguir, a suspensão foi filtrada e o resíduo foi lavado com água destilada livre de $\mathrm{CO}_{2}$. O ácido acético produzido pela reação do acetato com o $\mathrm{H}^{+}$do grupo carboxílico foi titulado com $\mathrm{NaOH} 0,1 \mathrm{~mol} \mathrm{~L}^{-1}$, previamente padronizado com biftalato de $\mathrm{K}$ até $\mathrm{pH}$ 9,8 . 
Quadro 1. Propriedades químicas do solo e dos ácidos húmicos estudados

\begin{tabular}{|c|c|c|c|c|c|c|c|c|c|c|c|c|c|c|}
\hline Tratamento $^{(1)}$ & Camada & $\mathrm{pH}$ & $\mathbf{H}+\mathbf{A l}$ & $\mathrm{t}$ & $\mathbf{T}$ & V & PCLPZ & EC & EAH & $\mathrm{C}-\mathrm{COOH}$ & $\mathrm{C}-\mathrm{OH}$ & C-Ar & IF & CRLS \\
\hline \multicolumn{3}{|c|}{$\mathrm{m}$} & \multicolumn{3}{|c|}{$-\mathrm{cmol}_{\mathrm{c}} \mathrm{kg}^{-1}$} & \multicolumn{2}{|l|}{$\%$} & \multicolumn{2}{|c|}{$-\mathrm{tha}^{-1}-$} & \multicolumn{3}{|c|}{$\longrightarrow \mathrm{ppm}$} & u.a. & $\left(x 10^{17}\right)$ spins $g^{-1}$ \\
\hline $\mathrm{CC}$ & $0-0,20$ & 6,7 & 2,2 & 12,8 & 15,0 & 85 & 3,1 & 59,36 & 2,56 & 34 & 13 & 9 & 52 & 2,49 \\
\hline $\mathrm{CC}$ & $0,20-0,40$ & 6,8 & 1,7 & 12,8 & 14,5 & 88 & 3,7 & 56,40 & 2,30 & 33 & 10 & 9 & 47 & 1,08 \\
\hline $\mathrm{CQ}$ & $0-0,20$ & 6,5 & 2,3 & 8,1 & 10,4 & 78 & 3,7 & 36,12 & 0,99 & 10 & 11 & 12 & 24 & 0,34 \\
\hline $\mathrm{CQ}$ & $0,20-0,40$ & 6,6 & 2,5 & 7,6 & 10,1 & 75 & 4,2 & 37,27 & 0,47 & 9 & 9 & 12 & 20 & 0,52 \\
\hline $\mathrm{CV}$ & $0-0,20$ & 5,9 & 3,7 & 8,4 & 12,2 & 69 & 3,3 & 52,00 & 1,11 & 20 & 9 & 7 & 31 & 1,30 \\
\hline $\mathrm{CV}$ & $0,20-0,40$ & 6,3 & 3,0 & 7,5 & 10,5 & 72 & 3,0 & 33,77 & 0,59 & 14 & 10 & 10 & 26 & 0,83 \\
\hline SV & $0-0,20$ & 6,0 & 3,2 & 7,8 & 11,0 & 71 & 3,5 & 48,21 & 0,86 & 19 & 10 & 4 & 30 & 0,36 \\
\hline SV & $0,20-0,40$ & 6,1 & 2,1 & 7,3 & 9,4 & 77 & 3,3 & 32,52 & 0,39 & 13 & 11 & 8 & 26 & 0,38 \\
\hline
\end{tabular}

(1) Tratamento: CC, CQ, CV e SV = manejo em cana crua, cana queimada, com vinhaça e sem vinhaça, respectivamente. Solo pH: relação solo:água igual a 1:2,5; $\mathrm{H}+\mathrm{Al}$ : acidez potencial extraída com acetato de amônio 0,5 mol L ${ }^{-1}$ a pH 7; t e T: CTC efetiva e potencial; V: saturação por bases; PCPLZ: ponto de carga protônica líquida zero; EC e EAH: estoque de C e da fração ácidos húmicos; Ácidos húmicos - C-COOH, C-OH e C-Ar: C em grupos carboxílicos, fenólicos e aromáticos por ressonância magnética nuclear do ${ }^{13} \mathrm{C}$; IF: intensidade de fluorescência (u.a.: unidade arbitrária); CRLS: concentração de radicais livres do tipo semiquinonas por ressonância paramagnética eletrônica.

A concentração de grupos quinonas nos $\mathrm{AH}$ foi estimada pelo método da redução a hidroquinona, usando-se o cloreto de estanho (II) em meio alcalino como redutor, conforme Schnitzer \& Riffaldi (1972). Adicionaram-se ao frasco $20 \mathrm{mg}$ de $\mathrm{AH}, 0,2 \mathrm{~mL}$ de etanol e $10 \mathrm{~mL}$ de $\mathrm{NaOH} 0,1 \mathrm{~mol} \mathrm{~L}^{-1}$ sob agitação constante até à solubilização. Em seguida, foram adicionados $20 \mathrm{~mL}$ de $\mathrm{NaOH} 2,5 \mathrm{~mol} \mathrm{~L}^{-1}, 10 \mathrm{~mL}$ de $\mathrm{SnCl}_{2} 6 \mathrm{H}_{2} \mathrm{O} \quad 0,05 \mathrm{~mol} \mathrm{~L}^{-1}$, e o frasco foi fechado. Procedeu-se à reação por uma hora sob agitação constante e sob atmosfera inerte de $\mathrm{N}_{2}$. O excesso do redutor $\left(\mathrm{Sn}^{2+}\right)$ foi titulado potenciometricamente, usando-se o $\mathrm{K}_{2} \mathrm{Cr}_{2} \mathrm{O}_{7} 0,05 \mathrm{~mol} \mathrm{~L}^{-1}$.

Os $\mathrm{AH}$ isolados foram submetidos à derivação química por metilação e acetilação (Sachs et al., 2002). Os derivados acetilados foram obtidos após a reação com anidrido acético em presença de piridina sob agitação magnética contínua à temperatura ambiente, durante uma semana. O processo de metilação de $\mathrm{AH}$ foi realizado com diazometano $\left(\mathrm{CH}_{2} \mathrm{~N}_{2}\right)$, produzido a partir do reagente $\mathrm{N}$-metil-N-nitrosotolueno-psulfonamida em éter etílico, na presença de metanol. $\mathrm{O}$ procedimento de metilação dos $\mathrm{AH}$ foi repetido por três vezes e finalizado quando a incorporação do diazometano aos $\mathrm{AH}$ foi completada, indicada pela coloração amarela do $\mathrm{CH}_{2} \mathrm{~N}_{2}$. As reações de metilação e acetilação dos $\mathrm{AH}$ foram monitoradas por meio da espectroscopia na região do infravermelho com transformada de Fourier (IV-TF), entre as faixas de 400 a 4.000 número de ondas $\mathrm{cm}^{-1}$. Os espectros foram obtidos usando-se pastilhas com $1 \mathrm{mg}$ de $\mathrm{AH}$ em $100 \mathrm{mg}$ de KBr, em um aparelho Shimadzu 83000 (Stevenson, 1994). Considerou-se a reação satisfatória quando os espectros de IV-TF mostraram aumento de absorção em 1.735-1.742 cm-1 e em 2.927-2.942 cm-1, indicando que a metilação dos $\mathrm{AH}$ converteu grupos oxigenados em ésteres e que a acetilação "bloqueou" os grupos fenólicos e quinonas livres.

\section{Titulações redox}

As titulações redox consistiram da oxidação dos $\mathrm{AH}$ estudados com $\mathrm{I}_{2}$ (iodimetria), sob atmosfera inerte (Struyk \& Sposito, 2001). Utilizou-se um frasco hermético (Figura 1), mantendo-se a temperatura do titulado a $25^{\circ} \mathrm{C}$ por meio de fluxo de água contínuo proporcionado pelo acoplamento do frasco de titulação a um equipamento de "banho termostatizado" (Microquímica ${ }^{\circledR}$ Banho MQBCT 99-20).

Preparou-se a tampa do frasco para acomodar um eletrodo combinado para $\mathrm{pH}$ (pHmetro Quimis Q400A) e um eletrodo redox combinado (Analyser 6A05-GK $\mathrm{AgCl}, \mathrm{Ag}|| \mathrm{Pt}$ ), além de um tubo para entrada de gás argônio (Ar) e uma microbureta de $2 \mathrm{~mL}$ (Gilmont ${ }^{\mathbb{B}}$ GS-1200-A, com subdivisões de $2 \mu \mathrm{L}$ ), usando-se silicone para a vedação.

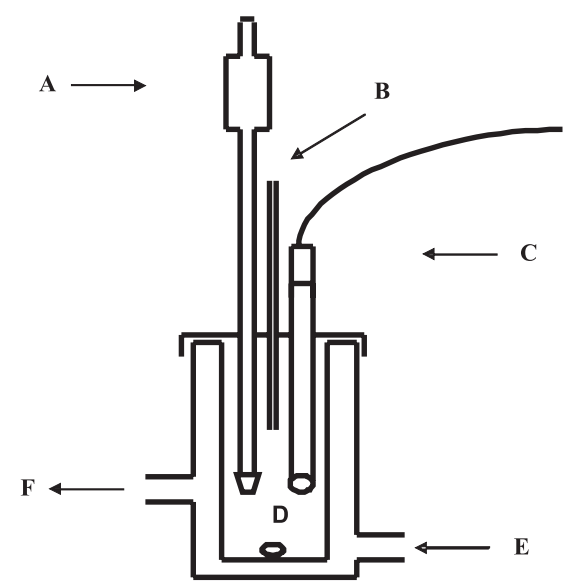

Figura 1. Esquema do equipamento de titulação redox. A: microbureta; B: tubo para entrada de Ar; C: eletrodo de Pt combinado (referência AgCl, Ag); D: agitador magnético; E e F: entrada e saída de água a $25^{\circ} \mathrm{C}$, respectivamente. 
No preparo do titulado, adicionaram-se ao frasco de titulação $125 \mathrm{~mL}$ de solução-tampão fosfato $\left(\mathrm{Na}_{2} \mathrm{HPO}_{4} 0,05 \mathrm{~mol} \mathrm{~L}^{-1}\right.$ e $\left.\mathrm{NaH}_{2} \mathrm{PO}_{4} \mathrm{H}_{2} \mathrm{O} 0,05 \mathrm{~mol} \mathrm{~L}^{-1}\right)$ com $\mathrm{KCl} 0,05 \mathrm{~mol} \mathrm{~L}^{-1} \mathrm{e}$, em seguida, ajustou-se o $\mathrm{pH}$ com $\mathrm{HCl}$ ou $\mathrm{NaOH}$ para pH 5,00 ou 7,00. A solução foi agitada magneticamente e borbulhada com orgânico (Ar) por 60 min para a depleção de $\mathrm{O}_{2}$. Acrescentouse AH à solução com vista em obter uma concentração em suspensão de $50 \mathrm{mg} \mathrm{L}^{-1}$ (6,25 $\mathrm{mg}$ AH suspensos em $125 \mathrm{~mL}$ solução-tampão fosfato $0,05 \mathrm{~mol} \mathrm{~L}^{-1} \mathrm{com}$ $\mathrm{KCl}$ 0,05 $\mathrm{mol} \mathrm{L}^{-1}$ ). Novamente, agitou-se e borbulhouse a suspensão com Ar durante $30 \mathrm{~min}$. Em seguida, ajustou-se o $\mathrm{pH}$ da suspensão para 5 ou $7 \mathrm{com} \mathrm{HCl}$ ou $\mathrm{NaOH}$ para o início da titulação.

Para cada titulação, calibrou-se o pHmetro utilizando soluções-tampão a pH 4 e a 7 . O eletrodo redox foi calibrado com solução-tampão redox férricoferrosa, preparada com sulfato ferroso amoniacal $0,100 \mathrm{~mol} \mathrm{~L}^{-1}$, sulfato férrico amoniacal $0,100 \mathrm{~mol} \mathrm{~L}^{-1}$ $\mathrm{e}_{2} \mathrm{SO}_{4} 1,00 \mathrm{~mol} \mathrm{~L}^{-1}$. A leitura da força eletromotriz da cela unitária (f.e. $\mathrm{m}_{\text {cela }}$ ) em $439 \pm 2 \mathrm{mV}$ indicou o correto funcionamento do eletrodo (Light, 1972).

Padronizou-se a solução de $\mathrm{I}_{2}$, para cada titulação, utilizando tiossulfato de sódio de acordo com as reações propostas por Bard et al. (1985):

$$
\begin{array}{lr}
2 \mathrm{~S}_{2} \mathrm{O}_{3}{ }^{2-}(\mathrm{aq})=\mathrm{S}_{4} \mathrm{O}_{6}{ }^{2-}(\mathrm{aq})+2 \mathrm{e}^{-} & \mathrm{E}_{\mathrm{H}}{ }^{\circ}=-0,080 \mathrm{~V}(1) \\
\mathrm{I}_{2}(\mathrm{aq})+2 \mathrm{e}^{-}=2 \mathrm{I}-(\mathrm{aq}) & \mathrm{E}_{\mathrm{H}^{\circ}}=0,621 \mathrm{~V}(2) \\
\hline 2 \mathrm{~S}_{2} \mathrm{O}_{3}{ }^{2-}(\mathrm{aq})+\mathrm{I}_{2}(\mathrm{aq})=\mathrm{S}_{4} \mathrm{O}_{6}{ }^{2-}(\mathrm{aq})+2 \mathrm{I}-(\mathrm{aq}) & \mathrm{E}_{\mathrm{H}}{ }^{\circ}=0,541 \mathrm{~V}(3)
\end{array}
$$

Nas titulações, utilizaram-se incrementos de $2 \mu \mathrm{L}$ de solução $0,025 \mathrm{~mol} \mathrm{~kg}^{-1} \mathrm{de} \mathrm{I}_{2}\left(\mathrm{~mol} \mathrm{~kg} \mathrm{~kg}^{-1}=\right.$ molinidade, ou seja, o número de mols de $\mathrm{I}_{2}$ por kg de solução) com densidade conhecida e, após cada incremento de titulante, a solução foi agitada e borbulhada com Ar por mais $30 \mathrm{~s}$ para facilitar a homogeneização do sistema. Em seguida, registrou-se a leitura da f.e.m. cela após cada adição de titulante, sempre quando a variação no aparelho foi inferior ou igual a

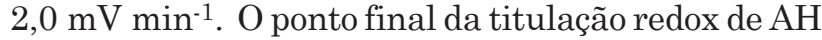
foi atingido quando valores constantes de f.e.m.cela foram registrados após a adição de oxidante.

As curvas de titulação redox foram obtidas, relacionando-se num gráfico os valores de f.e.m.cela (eixo das ordenadas) de acordo com a $\mathrm{COx}$ dos $\mathrm{AH}$ (eixo das abscissas). $\mathrm{O}$ valor da $\mathrm{COx}$ foi determinado pelo número de mols de $\mathrm{I}_{2}(\mathrm{aq})$ reduzidos a I- (aq) na reação por unidade de massa de $\mathrm{AH}\left(\mathrm{mol}_{\mathrm{c}} \mathrm{kg}^{-1}\right)$, usando-se a equação (Struyk \& Sposito, 2001):

mol $_{\mathrm{c}} \mathrm{kg}^{-1}=\frac{2 \text { mol }_{\mathrm{c}}}{\operatorname{mol~I}_{2}} \times \frac{\text { Densidade da Solução de } \mathrm{I}_{2} \times \text { Molinidade da Solução de } \mathrm{I}_{2}}{\text { Massa de AH }}$

Determinou-se o ponto de inflexão de cada curva de titulação redox obtendo-se a segunda derivada da função. No ponto de inflexão, a f.e.m. cela $_{\text {foi estimada }}$ por interpolação (Struyk \& Sposito, 2001). Adicionalmente, foram efetuadas titulações de amostras em branco, sem adição de $\mathrm{AH}$.

\section{Análise dos dados das titulações redox}

Analisaram-se os dados obtidos com a titulação redox dos $\mathrm{AH}$ de acordo com estudo desenvolvido inicialmente por Struyk \& Sposito (2001). Os valores de força eletromotriz (f.e.m.cela), medidos durante as titulações redox, foram relacionados com o potencial formal do eletrodo ( ${ }^{\mathrm{F}} \mathrm{E}_{\mathrm{H}}$ ) de acordo com a equação:

$$
\text { f.e.m.cela }=\mathrm{F}_{\mathrm{H}}\left(\mathrm{HA}_{\mathrm{Ox}}, \mathrm{HA}_{\mathrm{Red}}\right)-\mathrm{E}_{\mathrm{H}}^{\circ}(\mathrm{AgCl}, \mathrm{Ag})
$$

em que $\mathrm{E}_{\mathrm{H}}{ }^{\circ}(\mathrm{AgCl}, \mathrm{Ag})=222 \mathrm{mV}$ é o potencial padrão do eletrodo de referência $\left(\mathrm{AgCl}, \mathrm{Ag}\right.$ a $\left.25^{\circ} \mathrm{C}\right)$ relativo ao eletrodo padrão de hidrogênio e ${ }^{\mathrm{F}} \mathrm{E}_{\mathrm{H}}\left(\mathrm{HA}_{\mathrm{ox}}, \mathrm{HA}_{\text {red }}\right)$ é o potencial formal de eletrodo da meia-reação $\mathrm{AH}_{\mathrm{Ox}}$, $\mathrm{AH}_{\text {Red }}$, que correspondem, respectivamente, à forma oxidada e reduzida de $\mathrm{AH}$, de acordo com a semi-reação de redução:

$$
\mathrm{AH}_{\mathrm{Ox}}+\mathrm{ne}^{-}+\mathrm{q} \mathrm{H}^{+}=\mathrm{AH}_{\text {Red }}
$$

No ponto de inflexão (Stumm \& Morgan, 1996; Struyk \& Sposito, 2001):

$$
\begin{aligned}
& \text { f.e.m. } \text {.cela }={ }^{\mathrm{F}^{\mathrm{E}}}{ }_{\mathrm{H}}\left(\mathrm{AH}_{\mathrm{Ox}}, \mathrm{AH}_{\mathrm{Red}}\right)- \\
& (\mathrm{q} / \mathrm{n}) 0,05916 \mathrm{pH}-\mathrm{E}^{\circ}{ }_{\mathrm{H}}(\mathrm{AgCl}, \mathrm{Ag})
\end{aligned}
$$

na qual ${ }^{\mathrm{F}} \mathrm{E}_{\mathrm{H}}^{\mathrm{o}}\left(\mathrm{AH}_{\mathrm{ox}}, \mathrm{AH}_{\text {red }}\right)$ indica o potencial formal padrão do eletrodo de $\mathrm{AH}$.

A relação $\mathrm{q} / \mathrm{n}$ indica o número de mols de prótons (q) por mols de elétrons (n) transferidos durante a oxidação de $1 \mathrm{~mol}$ de AH. Foi calculada utilizando-se os valores da f.e.m.cela, obtidos para dois valores de $\mathrm{pH}(5,0$ e 7,0$)$, por meio da equação:

$$
-\frac{\mathrm{q}}{\mathrm{n}}=\frac{\frac{\Delta \text { f.e.m. }}{0,05916}}{\Delta \mathrm{pH}}
$$

em que $\Delta$ f.e.m. é a variação da f.e.m.cela por unidade log de variação do $\mathrm{pH}(\Delta \mathrm{pH})$ no ponto de inflexão da titulação.

De posse dos valores da relação $\mathrm{q} / \mathrm{n}$ e da f.e.m. cela no ponto de inflexão, calculou-se o ${ }^{\mathrm{F}} \mathrm{E}_{\mathrm{H}}^{\mathrm{o}}$ para a meia reação redox $\left(\mathrm{AH}_{\mathrm{OX}}, \mathrm{AH}_{\mathrm{Red}}\right)$, usando-se a equação 7 .

$\mathrm{O}^{\mathrm{F}} \mathrm{E}_{\mathrm{H}}{ }^{\mathrm{o}}$ de cada amostra de $\mathrm{AH}$ foi convertido na constante de equilíbrio condicional usando-se a seguinte relação (Stumm \& Morgan, 1996):

$$
\log \mathrm{K}_{\mathrm{c}}=\frac{{ }^{\mathrm{F}} \mathrm{E}_{\mathrm{H}}^{\mathrm{o}}}{0,05916}
$$

na qual $\mathrm{K}_{\mathrm{c}}$ é a constante de equilíbrio condicional, a $298 \mathrm{~K}$, correspondendo à reação de redução genérica para um elétron transferido (Struyk \& Sposito, 2001):

$$
\mathrm{m} \mathrm{Ox}+(\mathrm{q} / \mathrm{n}) \mathrm{H}^{+}+\mathrm{e}^{-}=\mathrm{pRed}
$$

$\mathrm{O} \mathrm{pe}_{\mathrm{c}}$ para o par redox $\mathrm{AH}_{\mathrm{Ox}}, \mathrm{AH}_{\mathrm{Red}}$ foi obtido conforme a equação (Stumm \& Morgan, 1996):

$$
\mathrm{pe}_{\mathrm{c}}=\log \mathrm{K}_{\mathrm{c}}-(\mathrm{q} / \mathrm{n}) \mathrm{pH}
$$




\section{Análises estatísticas}

Estimaram-se o erro-padrão da média para cada variável experimental e a correlação linear de Pearson entre a capacidade de oxidação e a concentração de grupos funcionais dos $\mathrm{AH}$. As estimativas dos coeficientes de correlação foram submetidas ao teste F, a 1 e $5 \%$ de significância (Steel \& Torrie, 1960).

\section{RESULTADOS E DISCUSSÃO}

As curvas de titulação potenciométrica dos $\mathrm{AH}$ foram semelhantes às obtidas por Struyk \& Sposito (2001), usando três amostras-padrão da IHSS, e às apresentadas por Baldotto et al. (2007), usando amostras isoladas de adubos orgânicos. Uma das curvas de titulação redox iodimétrica dos $\mathrm{AH}$ encontrase na figura 2.

Os valores de f.e.m. cela e de COx para as titulações redox realizadas a $\mathrm{pH} 5$ e 7 , bem como os resultados dos cálculos de ${ }^{\mathrm{F}} \mathrm{E}_{\mathrm{H}}{ }^{\circ}, \log \mathrm{K}_{\mathrm{c}}, \mathrm{pe}_{\mathrm{c}}$, encontram-se no quadro 2.

Em ambos os trabalhos, foram usadas soluções aquosas de $\mathrm{I}_{2}$ como oxidante. Esses $\mathrm{AH}$ apresentaram ${ }^{{ }^{2}} \mathrm{E}_{\mathrm{H}}{ }^{\circ}$ e $\log \mathrm{K}_{\mathrm{c}}$ análogos aos de compostos quinonas (Helburn \& MacCarthy, 1994). Contudo, essas duas substâncias diferenciaram-se pela mais ampla relação $\mathrm{q} / \mathrm{n}$ verificada nos grupos quinona $(1,00)$ em relação aos $\mathrm{AH}(0,33)$, valores também verificados para os $\mathrm{AH}$ isolados dos solos sob cana-de-açúcar sob diferentes sistemas de manejo.

Uma comparação entre os valores de alguns pares redox comuns nos sistemas naturais, realizada por Struyk \& Sposito (2001) e por Baldotto et al. (2007), permitiu alocar o par redox $\mathrm{AH}_{\mathrm{Ox}}, \mathrm{AH}_{\mathrm{Red}}$ na "seqüência redox" indicada por Stumm \& Morgan (1996). Os AH

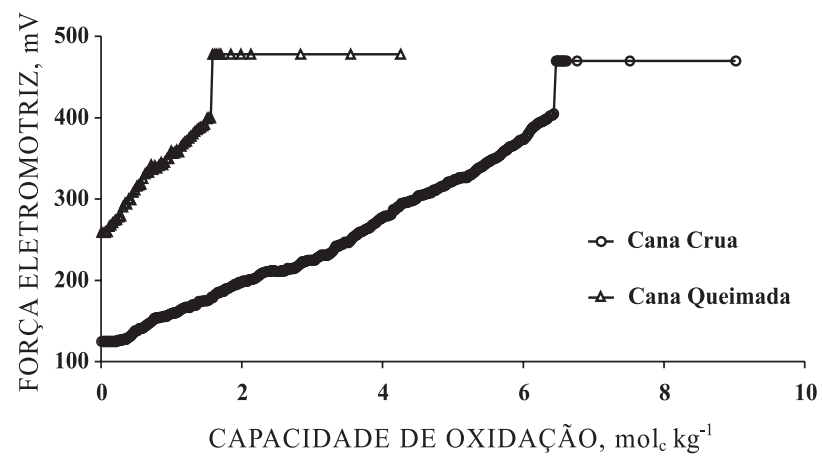

Figura 2. Titulações redox de $6,25 \mathrm{mg}$ de ácidos húmicos isolados da camada de 0-0,20 $\mathrm{m}$ de solo sob cana crua e sob cana queimada, suspensos

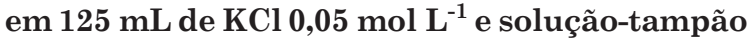
fosfato $0,05 \mathrm{~mol} \mathrm{~L}^{-1}$ a $\mathrm{pH} 7,00$. Os pontos de inflexão das curvas correspondem a f.e.m $\mathrm{mela}_{\text {a }}=$ $405 \pm 7 \mathrm{mV}$ e COx $=6,44 \pm 0,06 \mathrm{~mol}_{\mathrm{c}} \mathrm{kg}^{-1}$ para cana crua e a f.e. $m_{\text {cela }}=406 \pm 5 \mathrm{mV}$ e COx $=1,64 \pm$ $0,08 \mathrm{~mol}_{\mathrm{c}} \mathrm{kg}^{-1}$ para cana queimada.

podem reduzir $\mathrm{Hg}$ (II) e $\mathrm{Mn}$ (IV), conforme observaram Alberts et al. (1974) e Sunda \& Kieber (1994), respectivamente, incubando tais íons metálicos com bactérias redutoras e AH. Nos sistemas naturais, as implicações ambientais e biogeoquímicas das reações redox que envolvem os $\mathrm{AH}$ e os produtos formados ainda não se encontram bem descritas (Stumm \& Morgan, 1996; Struyk \& Sposito, 2001; Baldotto et al., 2007).

Baldotto et al. (2007) introduziram os dados médios dos $\mathrm{AH}$ estudados $\left(\mathrm{F}_{\mathrm{H}}{ }^{\circ}=0,78 \mathrm{~V}\right)$ no diagrama pe vs pH, adaptado de Bartlett \& James (1993), e obtiveram informações sobre a atividade das suas espécies $\left(\mathrm{AH}_{\mathrm{Ox}, \mathrm{Red}}\right)$ nos sistemas naturais. Assim, relataram que os AH poderiam atuar como doadores de elétrons nas reações

Quadro 2. Capacidade de oxidação $(\mathrm{COx})$ e potencial formal padrão do eletrodo $\left({ }^{\mathrm{F}} \mathrm{E}_{\mathrm{H}}^{\mathrm{o}}\right)$ dos ácidos húmicos

\begin{tabular}{|c|c|c|c|c|c|c|c|}
\hline \multirow{2}{*}{ Tratamento ${ }^{(1)}$} & \multirow{2}{*}{ Camada } & & & \multicolumn{2}{|c|}{ f.e.m $m_{\text {cela }}$} & \multirow{2}{*}{$\mathbf{F E}_{\mathrm{H}}^{\circ}$} & \multirow{2}{*}{$\log K_{c}$} \\
\hline & & pH 5 & pH 7 & pH 5 & pH 7 & & \\
\hline
\end{tabular}

\begin{tabular}{|c|c|c|c|c|c|c|c|c|}
\hline \multirow[b]{2}{*}{$\mathrm{CC}$} & \multirow{2}{*}{$\begin{array}{r}\mathrm{m} \\
0-0,20\end{array}$} & \multicolumn{2}{|c|}{$\operatorname{mol}_{\mathrm{c}} \mathrm{kg}^{-1} \longrightarrow$} & \multicolumn{2}{|c|}{$-\mathrm{mV}$} & \multicolumn{2}{|l|}{ V } & \multirow[b]{2}{*}{10,6} \\
\hline & & $3,44 \pm 0,06$ & $6,44 \pm 0,06$ & $444 \pm 4$ & $405 \pm 7$ & 0,764 & 12,9 & \\
\hline $\mathrm{CC}$ & $0,20-0,40$ & $2,02 \pm 0,04$ & $3,42 \pm 0,04$ & $440 \pm 4$ & $401 \pm 3$ & 0,760 & 12,8 & 10,5 \\
\hline $\mathrm{CQ}$ & $0-0,20$ & $1,01 \pm 0,05$ & $1,64 \pm 0,08$ & $445 \pm 3$ & $406 \pm 5$ & 0,765 & 12,9 & 10,6 \\
\hline $\mathrm{CQ}$ & $0,20-0,40$ & $1,22 \pm 0,05$ & $3,02 \pm 0,07$ & $451 \pm 6$ & $410 \pm 7$ & 0,776 & 13,1 & 10,7 \\
\hline $\mathrm{CV}$ & $0-0,20$ & $2,68 \pm 0,06$ & $4,99 \pm 0,06$ & $457 \pm 3$ & $417 \pm 6$ & 0,779 & 13,2 & 10,8 \\
\hline $\mathrm{CV}$ & $0,20-0,40$ & $2,00 \pm 0,09$ & $3,42 \pm 0,05$ & $449 \pm 5$ & $409 \pm 7$ & 0,771 & 13,0 & 10,7 \\
\hline SV & $0-0,20$ & $1,60 \pm 0,09$ & $2,37 \pm 0,06$ & $451 \pm 5$ & $412 \pm 6$ & 0,771 & 13,0 & 10,7 \\
\hline SV & $0,20-0,40$ & $1,28 \pm 0,08$ & $2,19 \pm 0,08$ & $453 \pm 7$ & $413 \pm 4$ & 0,775 & 13,1 & 10,7 \\
\hline
\end{tabular}

(1) Tratamento: CC, CQ, CV e SV = manejo em cana crua, cana queimada, com vinhaça e sem vinhaça, respectivamente. As médias dos valores determinados experimentalmente para: fem cela $_{2}=$ força eletromotriz da cela unitária; $\mathrm{k}_{\mathrm{c}}=$ constante condicional de equilíbrio; $\mathrm{pe}_{\mathrm{c}}=$ inverso do log da atividade de elétrons estão acompanhadas pelo seu erro-padrão. As demais variáveis foram calculadas, como descrito na seção Material e Métodos. 
redox em sistemas naturais. O íon $\mathrm{Fe}^{3+}$ aquoso seria uma espécie termodinamicamente favorável à redução por $\mathrm{AH}$, embora a espécie iônica $\mathrm{Fe}(\mathrm{OH})_{2}{ }^{+}$poderia ser predominante em solução a pH entre 5 e 7 , valores utilizados nas titulações redox. Como o par redox $\mathrm{Fe}^{2+}$, $\mathrm{FeOOH}$ está abaixo do par redox $\mathrm{AH}_{\mathrm{Ox} \text {, Red }}$ entre os valores de pH 5 e 7, as espécies $\mathrm{FeOOH}$ e $\mathrm{AH}_{\text {Red }}$ poderiam predominar no sistema. Segundo Lovley et al. (1996, 1998) e Scott et al. (1998), os AH poderiam mediar a redução microbiana de Fe(III) a partir da fase sólida, como, por exemplo, a espécie FeOOH e, sendo assim, é possível que, após a redução microbiana, os AH apresentem potencial abaixo daqueles determinados, na ausência de microrganismos, por Struyk \& Sposito (2001), Baldotto et al. (2007) e no presente estudo.

Os valores da $\mathrm{COx}$ dos $\mathrm{AH}$ variaram de 1,01 a $3,44 \mathrm{~mol}_{\mathrm{c}} \mathrm{kg}^{-1}$ a pH 5 e de 1,64 a 6,44 $\mathrm{mol}_{\mathrm{c}} \mathrm{kg}^{-1}$ a $\mathrm{pH} 7,0$. Incrementos da $\mathrm{COx}$ com o aumento do $\mathrm{pH}$ foram descritos em outros trabalhos e podem ser entendidos de acordo com a equação 6 . Struyk \& Sposito (2001) determinaram valores de COx entre 1,09 e $6,50 \mathrm{~mol}_{\mathrm{c}} \mathrm{kg}^{-1}$ a $\mathrm{pH} 5$ e variando de 3,30 até 11,50 a pH 7 para AH isolados de amostras-padrão da IHSS. Baldotto et al. (2007), para AH obtidos de adubos orgânicos, verificaram COx de 3,88 a 4,39 a pH 5 e de 5,35 a 7,89 a pH 7. Matthiessen (1995), em amostras de $\mathrm{AH}$ sintético, encontrou valores médios de 7,5 mol $_{\mathrm{c}} \mathrm{kg}^{-1}$ a pH 5,0 e de 10,5 mol $_{\mathrm{c}} \mathrm{kg}^{-1}$ a pH 7,0. Tais variações são típicas dessas reações de redução que consomem prótons.

A COx dos $\mathrm{AH}$ permitiu contrastar os manejos da cana-de-açúcar estudados. Em média, os incrementos na $\mathrm{COx}$ dos $\mathrm{AH}$ obtidos da área de cana-de-açúcar manejada sem a queima na colheita sobre o solo cultivado com cana queimada foram de 240 e de $292 \%$ $(0-0,20 \mathrm{~m})$, e de 65 e de $13 \%(0,20-0,40 \mathrm{~m})$, para as titulações a $\mathrm{pH} 5$ e a $\mathrm{pH} 7$, respectivamente. De forma geral, o comportamento da COx dos $\mathrm{AH}$ observado para as áreas com deposição da palhada foi também verificado para aquelas que receberam vinhaça. Os incrementos na $\mathrm{COx}$ dos $\mathrm{AH}$ obtidos da área com vinhaça em relação aos da área sem essa prática foram de 67 e de $110 \%(0-0,20 \mathrm{~m})$, para as titulações a pH 5 e a pH 7, respectivamente. Nas amostras retiradas da profundidade de $0,20-0,40 \mathrm{~m}$, a variação foi de $56 \%$, para ambos os valores de $\mathrm{pH}$ da titulação.

\section{Grupos funcionais}

A correlação entre a $\mathrm{COx}$ (Quadro 2) e a concentração de grupos funcionais (Quadro 3) fenólicos (0,94 a pH 5 e 0,95 a pH 7) quinonas (0,96 a pH 5 e $0,95$ a $\mathrm{pH} 7)$ e semiquinonas $(0,95$ a $\mathrm{pH} 5$ e 0,96 a $\mathrm{pH} 7)$ dos $\mathrm{AH}$ foi positiva e significativa ( $\mathrm{p} \leq 0,01)$. Não se verificou correlação com a concentração de grupos carboxílicos.

Struyk \& Sposito (2001) não estimaram as correlações, mas sugeriram a relação entre a COx e a concentração de grupos funcionais tipo semiquinonas dos AH. Baldotto et al. (2007) verificaram tal relação entre a COx e os teores de grupos funcionais fenólicos, quinonas e semiquinonas. Scott et al. (1998) obtiveram correlações positivas e significativas entre a $\mathrm{COx}$ determinada na presença de microrganismos e a concentração de quinonas de AH. Todos os trabalhos supracitados também relataram a ausência de correlação entre a concentração de grupos carboxílicos e a COx dos AH. Helburn \& MacCarthy (1994) atribuíram os valores de COx obtidos nas titulações redox de $\mathrm{AH}$ sintéticos à presença de grupos fenólicos graças à estreita relação com a acidez fenólica dos $\mathrm{AH}$ estudados.

\section{Derivados químicos}

À semelhança do trabalho de Baldotto et al. (2007), as curvas de titulação redox foram drasticamente modificadas pela metilação e acetilação dos $\mathrm{AH}$ (Figura 3).

Quadro 3. Concentração de grupos funcionais nos ácidos húmicos

\begin{tabular}{|c|c|c|c|c|}
\hline \multirow{2}{*}{ Tratamento ${ }^{(1)}$} & \multirow{2}{*}{ Camada } & \multicolumn{2}{|c|}{ Grupos funcionais ${ }^{(2)}$} & \multirow{2}{*}{ Quinonas } \\
\hline & & Carboxílicos & Fenólicos & \\
\hline & $\mathrm{m}$ & 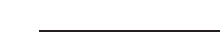 & $-\mathrm{mol}_{\mathrm{c}} \mathrm{kg}^{-1}$ & \\
\hline $\mathrm{CC}$ & $0-0,20$ & $4,77 \pm 0,02$ & $2,70 \pm 0,02$ & $0,85 \pm 0,02$ \\
\hline $\mathrm{CC}$ & $0,20-0,40$ & $4,53 \pm 0,03$ & $1,29 \pm 0,01$ & $0,54 \pm 0,01$ \\
\hline $\mathrm{CQ}$ & $0-0,20$ & $2,07 \pm 0,03$ & $0,79 \pm 0,01$ & $0,43 \pm 0,01$ \\
\hline $\mathrm{CQ}$ & $0,20-0,40$ & $3,48 \pm 0,04$ & $0,95 \pm 0,02$ & $0,48 \pm 0,01$ \\
\hline $\mathrm{CV}$ & $0-0,20$ & $4,60 \pm 0,02$ & $2,62 \pm 0,02$ & $0,77 \pm 0,01$ \\
\hline $\mathrm{CV}$ & $0,20-0,40$ & $4,48 \pm 0,03$ & $1,44 \pm 0,02$ & $0,59 \pm 0,02$ \\
\hline $\mathrm{SV}$ & $0-0,20$ & $2,15 \pm 0,03$ & $0,84 \pm 0,02$ & $0,51 \pm 0,01$ \\
\hline SV & $0,20-0,40$ & $3,49 \pm 0,03$ & $1,01 \pm 0,02$ & $0,55 \pm 0,01$ \\
\hline
\end{tabular}

(1) Tratamento: CC, CQ, CV e SV = manejo em cana crua, cana queimada, com vinhaça e sem vinhaça, respectivamente. ${ }^{(2)}$ As médias experimentais estão acompanhadas pelo seu erro-padrão. 


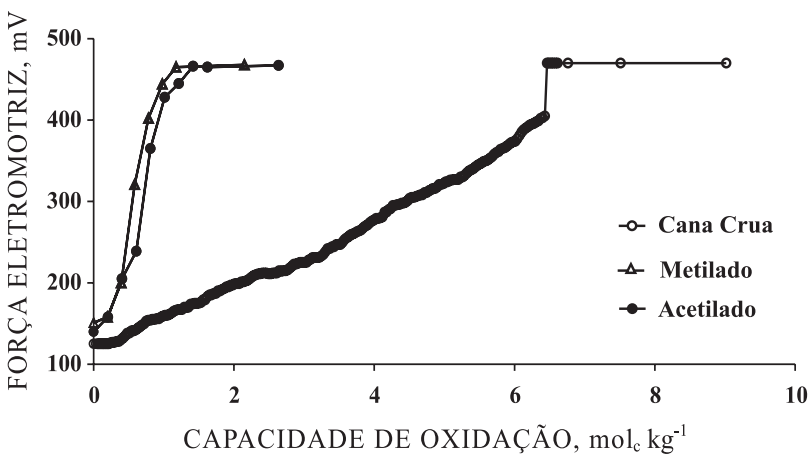

Figura 3. Titulações redox de $6,25 \mathrm{mg}$ de ácido húmico isolado da camada de $0-0,20 \mathrm{~m}$ do solo sob cana crua e de $6,25 \mathrm{mg}$ de seus derivados químicos obtidos por acetilação e por metilação, suspensos em $125 \mathrm{~mL}$ de $\mathrm{KCl} 0,05 \mathrm{~mol} \mathrm{~L}^{-1}$ e solução-tampão fosfato $0,05 \mathrm{~mol} \mathrm{~L}^{-1}$ a pH 7,00.

No presente estudo, esses processos também reduziram a $\mathrm{COx}$ dos $\mathrm{AH}$ para menos de $5 \%$, permitindo confirmar a importante contribuição dos grupos fenólicos e quinonas livres na transferência de elétrons nos $\mathrm{AH}$, visto que, à semelhança dos resultados de Baldotto et al. (2007), a metilação dos AH isolados dos solos sob cana-de-açúcar com diazometano $\left(\mathrm{CH}_{2} \mathrm{~N}_{2}\right)$ converteu grupos oxigenados ácidos em ésteres, que, no caso das SH são denominados humatos de metila $\left(\mathrm{R}-\mathrm{OCH}_{3}\right)$ e a acetilação dos grupos fenólicos e quinonas livres com anidrido acético em piridimina acetilaram grupos fenólicos e quinonas livres, ambas as reações "bloqueando" analogamente tais grupos doadores durante a reação de oxidação iodimétrica.

Dois mecanismos têm sido usados para explicar a alta correlação obtida entre a COx e as concentrações de grupos fenólicos, quinonas e semiquinonas dos $\mathrm{AH}$ : (a) a redução de grupos quinonas e fenólicos a semiquinonas, proposta por Scott et al. (1998), e (b) a oxidação iodimétrica dos complexos Fe-grupos fenólicos dos AH, gerando radicais livres semiquinonas, proposta por Struyk \& Sposito (2001). Observando a abrupta redução da COx pela metilação ou acetilação, pode-se concluir que o ${ }^{\mathrm{F}} \mathrm{E}_{\mathrm{H}}{ }^{\circ}$ do par redox $\mathrm{AH}_{\mathrm{Ox}}, \mathrm{AH}_{\mathrm{Red}}$ define o poder redutor dos $\mathrm{AH}$ e a sua $\mathrm{COx}$, a quantidade de elétrons transferíveis por grupos fenólicos, quinonas e semiquinonas, já presentes ou alterados durante a titulação. A relação direta entre a COx e a aromaticidade seletiva, ou seja, teores de grupos fenólicos e semiquinonas, indicadas, respectivamente, por ressonância magnética eletrônica da matéria orgânica do solo $\left({ }^{13} \mathrm{C} R M N\right)$ e por ressonância paramagnética de elétrons (RPE) apresentadas por Canellas et al. (2007) para essas mesmas áreas (Quadro 1), evidencia a participação desses grupos funcionais. Assim, o aumento concomitante do grau de condensação e da atividade redox dos $\mathrm{AH}$ revela a possibilidade de se estabelecerem modelos preditivos entre a $\mathrm{COx}$ e o grau de humificação dos AH.
Adicionalmente, a $\mathrm{COx}$ dos $\mathrm{AH}$ relacionou-se estreitamente $(0,88$ a pH 5 e 0,81 a pH $7, \mathrm{p} \leq 0,01)$ com a intensidade de fluorescência (IF) dos $\mathrm{AH}$ (Quadro 1). Milori et al. (2002) e Pérez et al. (2004) mostraram que o grau de humificação dos $\mathrm{AH}$ pôde ser estimado por meio de modelos preditivos entre a aromaticidade na forma de CRLS e a IF. Essa última propriedade advém de fluoróforos que absorvem e emitem luz e que estão associados a aspectos estruturais e eletroquímicos das substâncias químicas.

Os efeitos negativos do fogo, em comparação com à preservação da palha sobre o solo ou à adição de vinhaça, foram a brusca queda na quantidade de elétrons transferíveis pelos AH. Segundo Almendros et al. (2003) e González-Pérez et al. (2004), o efeito do fogo na MOS diminui as concentrações de grupos funcionais nos AH. No presente estudo, tais transformações diminuíram a concentração de grupos doadores de cargas nos $\mathrm{AH}$, diminuindo sua COx.

Os resultados indicam, também, para a área de cana queimada, que o uso do fogo durante longo período levou à menor COx na camada de 0,20-0,40 m, apesar de o efeito direto pelo aumento da temperatura ser bem menor que nas camadas superficiais. A movimentação de cinzas em profundidade, acumulandose e, posteriormente, fazendo parte da estabilização da matéria orgânica humificada na camada subsuperfícial, pode explicar a expressão de AH com menores concentrações de grupos funcionais e inferior atividade redox. Canellas et al. (2007), estudando essas mesmas amostras por $\mathrm{RMN}{ }^{13} \mathrm{C}$, indicaram aumento de aromaticidade seletiva (fenóis e semiquinonas) nos AH da camada de 0,20-0,40 cm em relação à camada superficial na área de cana queimada.

Canellas et al. (2003, 2007) observaram melhorias na qualidade da MOS, indicadas pelos $\mathrm{AH}$ isolados das áreas de cana crua e com vinhaça e que foram refletidas em aumento da CTC e diminuição do PCZ dos solos (Quadro 1). Os AH com maior $\mathrm{COx}$, por exemplo, foram obtidos dos solos com os menores valores de PCZ. A redução do PCZ com o aumento da $\mathrm{COx}$ deve-se à maior estabilização dos $\mathrm{AH}$ sob aporte constante de matéria orgânica nas áreas de cana crua. Siqueira et al. (1990a,b) e Peixoto (1997) encontraram redução do PCZ com a adição de materiais orgânicos no solo. Os efeitos da MOS no abaixamento do PCZ são devidos, principalmente, à adsorção de ânions orgânicos nos sítios de carga positiva dos minerais de argila. Vale ressaltar que o efeito da MOS no abaixamento do PCZ dos solos aumenta com o aumento do intemperismo e com a predominância de óxidos de Fe e Al na mineralogia do solo (Sposito, 1989; Siqueira et al., 1990a,b,c; Peixoto, 1997).

Finalmente, Canellas et al. $(2003,2007)$ e Busato et al. $(2004 a, b)$ verificaram significativos incrementos na disponibilidade e labilidade de nutrientes e na estabilidade e permanência das frações humificadas (aumentos dos estoques de $\mathrm{C}$ e de $\mathrm{AH}$ ) nos solos sob cana crua e com vinhaça em relação à cana queimada 
e sem a aplicação de vinhaça. Segundo esses autores, a disponibilidade de nutrientes correlacionou-se significativamente com o aumento da fração $\mathrm{AH}$. Nesse mesmo sentido, no presente estudo, a COx dos $\mathrm{AH}$ foi também incrementada, indicando a interligação entre os processos ecológicos que condicionam a estabilização do $\mathrm{C}$ em formas humificadas, as reações de oxidação e de redução da MOS, a disponibilidade de nutrientes, etc.

\section{CONCLUSÕES}

1. Os valores do ${ }^{\mathrm{F}} \mathrm{E}_{\mathrm{H}}{ }^{\circ}$ para a semi-reação de redução do par redox $\mathrm{AH}_{\mathrm{Ox}}, \mathrm{AH}_{\mathrm{Red}}$ foram semelhantes entre os AH estudados, sendo, em média de 0,770 V.

2. A COx dos $\mathrm{AH}$ variou com o $\mathrm{pH}$ do sistema e com o manejo da cana-de-açúcar. A COx observada para os AH ficou entre 1,01 e 3,44 mol $_{\mathrm{c}} \mathrm{kg}^{-1}$ a $\mathrm{pH} 5 \mathrm{e}$ variando de 1,64 a 6,44 mol $_{\mathrm{c}} \mathrm{kg}^{-1} \mathrm{a} \mathrm{pH}$ 7,0

3. Os AH obtidos em amostras das áreas com preservação da palhada e adição de vinhaça sobre o solo apresentaram não só maiores teores de grupos fenólicos, quinonas e semiquinonas, mas também $\mathrm{COx}$ mais elevada que aqueles isolados dos solos manejados sob cana queimada e sem vinhaça.

4. As reações de derivação química por metilação e acetilação reduziram drasticamente e de forma semelhante a COx dos $\mathrm{AH}$.

\section{AGRADECIMENTOS}

O presente trabalho contou com auxílio dos projetos: Matéria orgânica em sedimentos de estuários e águas costeiras sob influência de monocultivos de cana-de-açúcar na região norte fluminense (CNPq/ CTHidro Proc. 556160/2006-1/151856/2007-9), Instituto do Milênio Estuários - transferência de materiais na interface continente-oceano (CNPq Proc. 420050/2005-1), da Cooperação Brasil-Alemanha POLCAMAR: o impacto de poluentes da monocultura da cana-de-açúcar em estuários e águas costeiras do NE-E do Brasil - transporte, destino e estratégias de gerenciamento sustentável (CNPq/BMBF Proc. 590002/2005-8) e Eletroquímica de substâncias húmicas em sistemas tropicais (CNPq Proc. 4813/ 2004-9). Os autores agradecem aos professores Gabriel de Araújo Santos (UFRRJ), Jair Felipe Ramalho (UFRRJ) e Maurício Paulo Ferreira Fontes (UFV), pelas importantes sugestões ao trabalho e, ao professor José Carlos Sena Maia (UFF), pela ajuda na interpretação dos dados das titulações redox.

\section{LITERATURA CITADA}

ALBERTS, J.J.; SCHINDLER, J.E. \& MILLER, R.W. Elemental mercury evolution mediated by humic acid. Science, 184:895-896, 1974.
ALMENDROS, G.; KNICKER, H. \& GONZÁLEZ-VILA, F.J. Rearrangement of carbon and nitrogen forms in peat after progressive isothermal heating as determined by solid-state 13C- and 15N-NMR spectroscopies. Org. Geochem., 34:1559-1568, 2003.

BALDotTo, M.A.; CANELlas, L.P.; CANELA, M.C.; SIMÕES, M.L.; MARTIN-NETO, L.; FONTES, M.P.F. \& VELlosO, A.C.X. Propriedades redox e grupos funcionais de ácidos húmicos isolados de adubos orgânicos. R. Bras. Ci. Solo, 3:465-475, 2007.

BARD, A.; PARSON, R. \& JORDAN, J., eds. Standard potentials in aqueous solution. New York, Marcel Dekker, 1985. $185 \mathrm{p}$.

BARTLETT, R.J. \& JAMES, B.R. Redox chemistry of soils. Adv. Agron., 50:151-208, 1993.

BUDZIAK, C.R.; MAIA, C.M.B.F. \& MANGRICH, A.S. Transformações químicas da matéria orgânica durante a compostagem de resíduos da indústria madeireira. Química Nova, 27:399-403, 2004.

BUSATO, J.G.; CANELLAS, L.P. \& VELLOSO, A.C.X. Fósforo num Cambissolo cultivado com cana-de-açúcar por longo tempo. I - Fracionamento seqüencial. R. Bras. Ci. Solo, 29:935-944, 2005a.

BUSATO, J.G.; CANELLAS, L.P.; RUMJANEK, V.M. \& VELLOSO, A.C.X. Fósforo num Cambissolo cultivado com cana-de-açúcar por longo tempo. II - Análise de ácidos húmicos por RMN ${ }^{31}$ P. R. Bras. Ci. Solo, 29:945-953, 2005 b.

CANELLAS, L.P.; BALDOTTO, M.A.; BUSATO, J.G.; MARCIANO, C.R.; MENEZES, S.C.; SILVA, N.M.; RUMJANEK, V.M.; VELLOSO, A.C.X.; SIMÕES, M.L. \& MARTIN-NETO, L. Estoque e qualidade da matéria orgânica de um solo cultivado com cana-de-açúcar por longo tempo. R. Bras. Ci. Solo, 31:331-340, 2007.

CANELLAS, L.P.; VELLOSO, A.C.X.; MARCIANO, C.R.; RAMALHO, J.F.G.P.; RUMJANEK, V.M.; REZENDE, C.E. \& SANTOS, G.A. Propriedades químicas de um Cambissolo cultivado com cana-de-açúcar, com preservação do palhiço e adição de vinhaça por longo tempo. R. Bras. Ci. Solo, 27:935-944, 2003.

EMPRESA BRASILEIRA DE PESQUISA AGROPECUÁRIA EMBRAPA. Centro Nacional de Pesquisa de Solos. Sistema brasileiro de classificação de solos. Rio de Janeiro, Produção de Informação, 1999. 412p.

GONZÁLEZ-PÉREZ， J.A.; GONZÁLEZ-VILA， F.J.; ALMENDROS, G. \& KNICKER, H. The effect of fire on soil organic matter: A review. Environ. Internat., 30:855870, 2004.

HELBURN, R.S. \& MACCARTHY, P. Determination of some redox properties of humic acids by alkaline ferricyanide titration. Anal. Chem. Acta, 295:263-272, 1994.

INTERNATIONAL HUMIC SUBSTANCES SOCIETY - IHSS. Products. Disponível em: <http://www.ihss.gatech.edu/ products.html> Acesso em 01 Ago. 2007.

LAMEGO, A.R. O homem e o brejo. Rio de Janeiro, Conselho Nacional de Geografia. 1945. 244p. 
LIGHT, T.S. Standard solution for redox potential measurements. Anal. Chem., 44:1038-1039, 1972.

LOVLEY, D.R.; COATES, J.D.; BLUNT-HARRIS, E.L.; PHILLIPS, E.J.P. \& WOODWARD, J.C. Humic substances as electron acceptors for microbial respiration. Nature, 382:445-448, 1996.

LOVLEY, D.R.; WOODWARD, J.C.; BLUNT-HARRIS, E.L.; HAYES, L.; PHILLIPS, E. J.P. \& COATES, J.D. Humic substances as mediator for microbially-catalized metal reduction. Acta Hidrochim. Hydrobiol., 26:152-157, 1998.

MATTHIESSEN, A. Determining the redox capacity of humic substances as a function of $\mathrm{pH}$. Vom Wasser, 84:229-235, 1995.

MILORI, D.M.B.P.; MARTIN NETO, L.; BAYER, C.; MIELNICZUCK, J. \& BAGNATO, V.S. Humification degree of soil humic acids determined by fluorescence spectroscopy. Soil Sci., 167:739-749, 2002.

PEIXOTO, R.T.G. Matéria orgânica e dinâmica das cargas elétricas dos solos: Processos e conseqüências. In CONGRESSO BRASILEIRO DE CIÊNCIA DO SOLO, 26., Rio de Janeiro, 1997. Anais. Rio de Janeiro, Sociedade Brasileira de Ciência do Solo/Centro Nacional de Pesquisa em Solos da Empresa Brasileira de Pesquisa Agropecuária, 1997. CD-ROM

PÉREZ, M.G.; MARTIN-NETO, L.; SAAB, S.C.; NOVOTNY, E.H.; MILORI, D.M.B.P.; BAGNATO, V.S.; COLNAGO, L.A.; MELO, W.J. \& KNICKER, H. Characterization of humic acids from a Brazilian Oxisol under different tillage systems by EPR, 13C NMR, FTIR and fluorescence spectroscopy. Geoderma, 118:181-190, 2004.

PICCOLO, A. The supramolecular structure of humic substances. Soil Sci., 166:810-832, 2001.

RIVERO, C.; CHIRENGE, T.; MA, L.Q. \& MARTINEZ, G. Influence of compost on organic soil matter quality under tropical conditions. Geoderma, 123:355-361, 2004.

SACHS, S.; BUBNER, M.; SCHMEIDE, K.; CHOPPIN, G.R.; HEISE, K.H. \& BERNHARD, G. Carbon-13 NMR spectroscopy studies on chemically modified and unmodified synthetic and natural humic acids. Talanta, 57:999-1009, 2002.
SCHNITZER, M. \& GUPTA, U.C. Determination of acidity in soil organic matter. Soil Sci. Soc. Am. Proc., 29:274-277, 1965.

SCHNITZER, M. \& RIFFALDI, R. The determination of quinone groups in humic substances. Soil Sci. Soc. Am. Proc., 36:772-777, 1972.

SCOTT, D.T.; MCKNIGHT, D.M.; HARRIS, E.; KOLESAR, S. \& LOVLEY D. Quinone moieties act as electron aceptors in the reducting of humic substances by humics-reducting microorganisms. Environ. Sci. Technol., 32:2984-2989, 1998.

SIQUEIRA, C.; LEAL, J.R. \& VELLOSO, A.C.X. Eletroquímica de solos tropicais de carga variável: III. Erros na avaliação das cargas. R. Bras. Ci. Solo, 14:19-24, 1990c.

SIQUEIRA, C.; LEAL, J.R.; VELLOSO, A.C.X. \& SANTOS, G.A. Eletroquímica de solos tropicais de carga variável: I. Influência da matéria orgânica no tempo de equilíbrio pra a determinação das curvas de titulação potenciométrica. R. Bras. Ci. Solo, 14:7-11, 1990a.

SIQUEIRA, C.; LEAL, J.R.; VELLOSO, A.C.X. \& SANTOS, G.A. Eletroquímica de solos tropicais de carga variável: II. Quantificação do efeito da matéria orgânica sobre o ponto de carga zero. R. Bras. Ci. Solo, 14:13-18, 1990b.

SPOSITO, G. Chemistry of soil. New York, Oxford University Press, 1989. 277p.

STEEL, R.G.D. \& TORRIE, J.H. Principles and procedures of statistics with special reference to the biological science. New York, McGraw-Hill Book Company, 1960. 453p.

STEVENSON, F.J. Humus chemistry: Gensesis, composition, reaction. 2.ed. New York, John Wiley \& Sons, 1994. 496p.

STRUYK, Z. \& SPOSITO, G. Redox properties of standard humic acids. Geoderma, 102:329-346, 2001.

STUMM, W. \& MORGAN, J.J. Aquatic chemistry: Chemical equilibria and rates in natural waters. New York, WileyInterscience, 1996. 1022p.

SUNDA, W.G. \& KIEBER, D.J. Oxidation of humic substances by manganese oxides yields low-molecular-weight organic substrates. Nature, 367:62-65, 1994. 